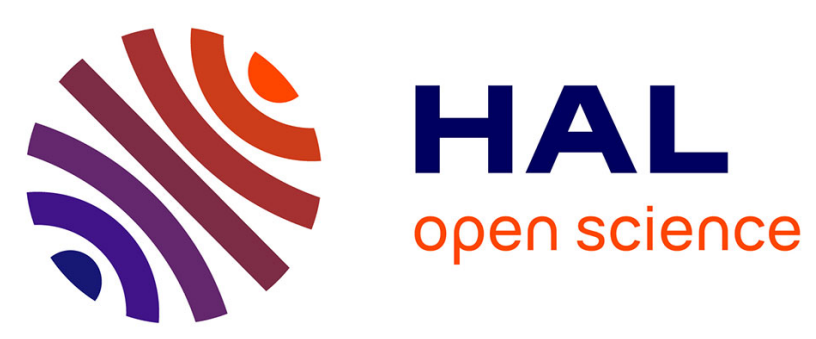

\title{
First-price sealed-bid auctions when bidders exhibit different attitudes toward risk
}

François Marechal, Pierre-Henri Morand

\section{To cite this version:}

François Marechal, Pierre-Henri Morand. First-price sealed-bid auctions when bidders exhibit different attitudes toward risk. Economics Letters, 2011, 113 (2), pp.108-111. hal-01313412

\section{HAL Id: hal-01313412 \\ https://hal.science/hal-01313412}

Submitted on 24 Nov 2017

HAL is a multi-disciplinary open access archive for the deposit and dissemination of scientific research documents, whether they are published or not. The documents may come from teaching and research institutions in France or abroad, or from public or private research centers.
L'archive ouverte pluridisciplinaire HAL, est destinée au dépôt et à la diffusion de documents scientifiques de niveau recherche, publiés ou non, émanant des établissements d'enseignement et de recherche français ou étrangers, des laboratoires publics ou privés. 


\title{
First-price sealed-bid auctions when bidders exhibit different attitudes toward risk
}

\author{
François Maréchal*and Pierre-Henri Morand ${ }^{\dagger}$ \\ May 2011
}

\begin{abstract}
When bidders have different risk aversion levels, we determine in a firstprice auction the asymmetric equilibrium strategies. We analyze the impact of asymmetric risk aversion levels on bidders' markups and on the expected revenue and allocative efficiency of the auction.
\end{abstract}

JEL classification: D44; D81.

Keywords: auction, asymmetric risk aversion.

*Corresponding author. CRESE, University of Franche-Comté, 30 avenue de l'observatoire, BP 1559, 25009 Besançon cedex, France. Tel: (+33) 3816667 47. francois.marechal@univfcomte.fr

${ }^{\dagger}$ CRESE, University of Franche-Comté (France). pierre-henri.morand@univ-fcomte.fr 


\section{Introduction}

The pioneering article of Vickrey (1961) in auction theory considered the case of risk neutral bidders. Since then the theory has been extended to risk averse buyers (see e.g. Milgrom and Weber (1982), Maskin and Riley (1984) or Matthews (1983)). These models assume that bidders exibit the same attitude toward risk. However, as noted by Campo (2009), experiments by Cox et al. (1985) and Goeree et al. (2002) shed light on the bidders' different attitudes toward risk. Similarly, Athey and Levin's (2001) empirical work on timber auctions suggests that risk aversion and its diversity cannot be ignored in auction models. To the best of our knowledge, the optimal bidding strategies of asymmetric bidders in terms of risk aversion have not been determined yet.

The study of asymmetric auctions within the standard informational framework of the Independent Private Value (IPV) model is a quite tedious problem (see e.g. Maskin and Riley (2000) for a study of the impact of asymmetric distributions of bidders' valuations on bidding strategies) since it involves solving a complex system of differential equations. This feature still remains with asymmetric bidders in terms of risk aversion. As for example, in their empirical works dealing with bidders having different attitudes toward risk, Guerre et al. (2000) and Campo (2009) study the model identification and estimation procedure without explicitly solving the equilibrium strategies. In order to solve these strategies explicitly and following Von Ungern-Sternberg (1991) (hereafter VUS), we choose to adopt a simpler informational paradigm which, in our opinion, does not constitute a great reduction in terms of realism as compared with the standard IPV model and enables us to derive bidders equilibrium strategies explicitly.

VUS's model actually combines the simplifying properties of the standard IPV and the common value models. Indeed, each bidder learns his own valuation with certainty and, as in the simplest version of the common value model, each bidder has no grounds for believing his own valuation estimate to be higher or lower on average than his competitor's valuations. Formally, VUS models this by assuming that the different bidders' valuations are independent drawings from a known distribution with an unknown mean. ${ }^{1}$ Since the bidders know the functional form of the distribution but are uncertain about the mean, they will infer this mean from their own private valuation.

VUS's model considers symmetric risk neutral bidders. In this paper, we extend the analysis of VUS to the case of risk averse buyers who exhibit different attitudes toward risk. In the next section, we outline the model. In section 3, we derive the asymmetric bidding equilibrium in the context of two risk averse bidders having different relative risk aversion levels. For a given bidder, we ana-

\footnotetext{
${ }^{1}$ This prior belief is depicted by Biais and Bossaerts (1998) as the "average opinion rule".
} 
lyze the impact on bidding aggressiveness of both his own risk aversion and that of his opponent. Namely, we provide conditions under which the less risk averse bidder reduces his markup although he becomes even less risk averse (while his opponent becomes more risk averse). Then, we analyze the impact of asymmetric risk aversion levels on allocative efficiency and expected revenue of the auction. Section 4 offers concluding remarks.

\section{The outline of the model}

We consider a two bidder first-price sealed-bid auction. Each bidder $i \in\{\alpha, \beta\}$ has a private valuation $v_{i}$. Both bidders are ex ante symmetric relative to the informational knowledge and believe that their private valuations are independently drawn from a distribution $F$ over $[\mu-a, \mu+a]$, where $a$ is common knowledge whereas the mean $\mu$ is unknown. Thus, when a bidder, say $\alpha$, learns his own valuation $v_{\alpha}$, he can infer that $\mu \in\left[v_{\alpha}-a, v_{\alpha}+a\right]$ according to the cumulative $F_{\mu}$ with corresponding density $f_{\mu}$. Since both bidders are ex ante symmetric relative to the informational knowledge, bidder $\alpha$ then infers that $v_{\beta} \in\left[v_{\alpha}-2 a, v_{\alpha}+2 a\right]$ according to the cumulative distribution $F_{\beta}$ with corresponding density $f_{\beta}$.

In order to highlight the impact of risk aversion on bidding strategies, let us assume that each bidder $i$ is characterized by a constant relative risk aversion (CRRA) utility function $U_{i}(x)=x^{\rho_{i}}$ (with $\left.0<\rho_{i} \leq 1\right)$, where $1-\rho_{i}$ is the CRRA parameter of bidder $i$ and $x$ is the bidder's income.

\section{The bidding strategies}

Within this informational framework, the fact that bidder $i$ privately knows his own valuation does not reveal anything to him about his relative position and so does not affect his probability of winning. Therefore, as in VUS's model, there is no reason why he should let his strategic mark-up depend on his own valuation. Hence, we can assume that each bidder's equilibrium bid has the following form

$$
B_{i}\left(v_{i}\right)=v_{i}-b_{i}
$$

where $b_{i}$ represents the mark-up (or profit) of bidder $i$. Consider firstly the case of bidder $\alpha$. Under a first-price sealed-bid auction, bidder $\alpha$ 's expected utility is given by

$$
E U_{\alpha}=\left(b_{\alpha}\right)^{\rho_{\alpha}} P\left(b_{\alpha}, b_{\beta}\right),
$$

where $P\left(b_{\alpha}, b_{\beta}\right)$ reflects his probability of winning when he chooses a strategic mark-up $b_{\alpha}$, while bidder $\beta$ chooses $b_{\beta}$. Differentiating (??) with respect to $b_{\alpha}$ 
yields the optimal mark-up for bidder $\alpha$

$$
\begin{aligned}
\frac{\partial E U_{\alpha}}{\partial b_{\alpha}} & =\left(b_{\alpha}\right)^{\rho_{\alpha}} P^{\prime}\left(b_{\alpha}, b_{\beta}\right)+\rho_{\alpha} b_{\alpha}{ }^{\left(\rho_{\alpha}-1\right)} P\left(b_{\alpha}, b_{\beta}\right)=0 \\
& \Leftrightarrow b_{\alpha} P^{\prime}\left(b_{\alpha}, b_{\beta}\right)+\rho_{\alpha} P\left(b_{\alpha}, b_{\beta}\right)=0 \\
& \Leftrightarrow b_{\alpha}=-\frac{\rho_{\alpha} P\left(b_{\alpha}, b_{\beta}\right)}{P^{\prime}\left(b_{\alpha}, b_{\beta}\right)} .
\end{aligned}
$$

Let us now derive the winning probability. Bidder $\alpha$ wins if $v_{\alpha}-b_{\alpha}>v_{\beta}-b_{\beta}$, i.e. if $v_{\beta}<v_{\alpha}-b_{\alpha}+b_{\beta}$, which occurs with probability $F_{\beta}\left(v_{\alpha}-b_{\alpha}+b_{\beta}\right)$. Then, ex ante, bidder $\alpha$ wins with probability $P$ (defined in expectation over the unknown mean $\mu$ ) such that

$$
P=\int_{\mu} F_{\beta}\left(v_{\alpha}-b_{\alpha}+b_{\beta}\right) f_{\mu}(\mu) d \mu .
$$

In order to provide an explicit form of the probability of winning, let us now consider the special case of a uniform distribution for $F$ (and therefore for $F_{j}$ and $F_{\mu}$ ). Then, we have

$$
P=\int_{\mu} \frac{1}{2 a}\left(\frac{\left.a-b_{\alpha}+b_{\beta}+v_{\alpha}-\mu\right)}{2 a}\right) d \mu .
$$

To compute this probability, assume ${ }^{2}$ e.g. that $b_{\alpha} \geq b_{j}$. Then $P \geq 0$ if $\mu \leq v_{\alpha}+a-b_{\alpha}+b_{\beta}$ and $P \leq 1$ if $\mu \geq v_{\alpha}-a-b_{\alpha}+b_{\beta}$. Since $b_{\alpha} \geq b_{\beta}$ and given that $\mu$ lies over the interval $\left[v_{\alpha}-a, v_{\alpha}+a\right]$, one thus has to integrate only over the interval $\left[v_{\alpha}-a, v_{\alpha}+a-b_{\alpha}+b_{\beta}\right]$. Then $\mathrm{P}$ becomes

$$
\begin{aligned}
P & =\int_{v_{\alpha}-a}^{v_{\alpha}+a-b_{\alpha}+b_{\beta}} \frac{1}{2 a}\left(\frac{\left.a-b_{\alpha}+b_{\beta}+v_{\alpha}-\mu\right)}{2 a}\right) d \mu \\
& =\frac{\left(2 a-b_{\alpha}+b_{\beta}\right)^{2}}{8 a^{2}} .
\end{aligned}
$$

Consider now the case of bidder $\beta$. Let $Q$ denote his winning probability. Obviously from (??), bidder $\beta$ chooses $b_{\beta}$ such that

$$
b_{\beta}=-\frac{\rho_{\beta} Q\left(b_{\alpha}, b_{\beta}\right)}{Q^{\prime}\left(b_{\alpha}, b_{\beta}\right)} .
$$

Bidder $\beta$ wins if $v_{\beta}-b_{\beta}>v_{\alpha}-b_{\alpha}$, i.e. if $v_{\alpha}<v_{\beta}-b_{\beta}+b_{\alpha}$, which occurs with probability $F_{\alpha}\left(v_{\beta}-b_{\beta}+b_{\alpha}\right)$. Then, ex ante, bidder $\beta$ wins with probability $Q$ such that

$$
Q=\int_{\mu} F_{\alpha}\left(v_{\beta}-b_{\beta}+b_{\alpha}\right) f_{\mu}(\mu) d \mu .
$$

\footnotetext{
${ }^{2}$ The same reasoning can be applied with $b_{\alpha} \leq b_{\beta}$.
} 
In the special case of a uniform distribution, we have

$$
Q=\int_{\mu} \frac{1}{2 a}\left(\frac{\left.a+b_{\alpha}-b_{\beta}+v_{\beta}-\mu\right)}{2 a}\right) d \mu .
$$

To compute this probability, we still assume that $b_{\alpha} \geq b_{\beta}$. Then $Q \geq 0$ if $\mu \leq a+b_{\alpha}-b_{\beta}+v_{\beta}$ and $Q \leq 1$ if $\mu \geq a+b_{\alpha}-b_{\beta}+v_{\beta}$. Since $b_{\alpha} \geq b_{\beta}$ and given that $\mu$ lies over the interval $\left[v_{\beta}-a, v_{\beta}+a\right]$, one thus has to integrate over the interval $\left[a+b_{\alpha}-b_{\beta}+v_{\beta}, v_{\beta}+a\right]$. Besides, when the true mean of the distribution lies in the interval $\left[v_{\beta}-a, v_{\beta}-a+b_{\alpha}-b_{\beta}\right]$ bidder $\beta$ is certain to win. Then $\mathrm{Q}$ becomes

$$
\begin{aligned}
Q & =\int_{a+b_{\alpha}-b_{\beta}+v_{\beta}}^{v_{\beta}+a} \frac{1}{2 a}\left(\frac{\left.a+b_{\alpha}-b_{\beta}+v_{\beta}-\mu\right)}{2 a}\right) d \mu+\int_{v_{\beta}-a}^{v_{\beta}-a+b_{\alpha}-b_{\beta}} \frac{1}{2 a} d \mu \\
& =\frac{4 a^{2}+4 a-\left(b_{\alpha}-b_{\beta}\right)^{2}}{8 a^{2}} .
\end{aligned}
$$

Given the values of $P$ and $Q$ and the first order conditions (??) and (??), we can derive the optimal strategic markups for both bidders ${ }^{3}$

$$
b_{\alpha}=\frac{a \rho_{\alpha}(1+A)}{2+\rho_{\alpha}+\rho_{\beta}} \text { and } b_{\beta}=\frac{a\left(\left(2+\rho_{\alpha}\right) A-2-\rho_{\alpha}-2 \rho_{\beta}\right)}{2+\rho_{\alpha}+\rho_{\beta}} .
$$

where $A=\sqrt{1+2 \rho_{\beta}\left(2+\rho_{\alpha}+\rho_{\beta}\right)}$. We can easily check that the bidding strategies are positive. Note that $b_{\alpha}$ and $b_{\beta}$ are increasing with respect to the uncertainty parameter $a$, which is consistent with intuition and conventional results in auction theory. ${ }^{4}$ In the following, we analyze the impact of asymmetric risk aversion levels on bidders' markups as well as on the expected revenue and allocative efficiency of the auction.

\subsection{The impact of risk aversion levels on bidders' markups}

We consider first the case of bidder $\alpha$. We can first compute

$$
\frac{\partial b_{\alpha}}{\partial \rho_{\alpha}}=\frac{a\left(2+\rho_{\beta}\left(9+2 \rho_{\alpha}^{2}+3 \rho_{\alpha}\left(2+\rho_{\beta}\right)+2 \rho_{\beta}\left(4+\rho_{\beta}\right)+3 A\right)\right)}{A\left(2+\rho_{\alpha}+\rho_{\beta}\right)^{2}}>0 .
$$

Thus, when bidder $\alpha$ becomes less risk averse, he increases his strategic mark-up. This is the direct effect of the modification of risk aversion on bidding strategy. ${ }^{5}$

\footnotetext{
${ }^{3}$ We check that the second order conditions are satisfied.

${ }^{4}$ See among others Klemperer (2001) or Krishna (2002).

${ }^{5}$ Raising one's bid slightly in a first-price sealed-bid is analogous to buying partial insurance.
} 
Besides, a bidder 's strategy is also affected by the modification of his opponent's risk aversion. We can compute

$$
\frac{\partial b_{\alpha}}{\partial \rho_{\beta}}=\frac{a \rho_{\alpha}\left(3-A+2 \rho_{\beta}+\rho_{\alpha}\left(4+\rho_{\alpha}+\rho_{\beta}\right)\right)}{A\left(2+\rho_{\alpha}+\rho_{\beta}\right)^{2}}>0,
$$

since $A \leq 3$. Actually, when his opponent is more risk averse, bidder $\alpha$ becomes more aggressive in order to reacts to his opponent's aggressiveness. This is the indirect effect. In order to alleviate notations and w.l.o.g. we can consider that $\rho_{\alpha}=\rho+\epsilon$ and $\rho_{\beta}=\rho-\epsilon$, where $\epsilon$ is a small positive real number $0<\epsilon<$ $\min (1-\rho, \rho)$. Then, the following proposition can be stated ${ }^{6}$

Proposition 1 For the most risk averse bidder, the direct effect dominates the indirect effect; for the less risk averse bidder, the indirect effect dominates the direct effect if $\epsilon>\frac{1+3 \rho+3 \rho^{2}+\sqrt{1+6 \rho+6 \rho^{2}}}{9(1+\rho)}$.

Thus, the strategy of bidder $\alpha$ is more affected by an increase of his opponent's risk aversion than by an increase of his own risk aversion when there is a large gap between both risk aversion levels. Then, bidder $\alpha$ is much more concerned by a change in $\rho_{\beta}$ than by a change in $\rho_{\alpha}$ when bidder $\beta$ is much more risk averse than him. It is worth noting that when the indirect effect dominates the direct effect, this means that bidder $\alpha$ becoming less risk averse, (while his opponent becomes more risk averse) reduces his markup.

\subsection{The impact of risk aversion levels on allocative efficiency}

Let us now analyze the impact of asymmetric risk aversion levels on the allocative efficiency of the auction. A traditional result on asymmetric auctions ${ }^{7}$ can be stated

Lemma 1 A first price sealed-bid auction with asymmetric risk averse bidders may preclude allocative efficiency.

Proof of lemma 1: Consider that $v_{\alpha}>v_{\beta}$ and $\rho_{\alpha}>\rho_{\beta}$. Then, the auction is efficient if $\alpha$ wins i.e. if $v_{\alpha}-b_{\alpha}>v_{\beta}-b_{\beta}$. However, substituting $b_{\alpha}$ and $b_{\beta}$ by their values, the latter inequality is not satisfied if

$$
\frac{v_{\alpha}-v_{\beta}}{a}<\frac{2\left(1+\rho_{\alpha}+\rho_{\beta}-A\right)}{2+\rho_{\alpha}+\rho_{\beta}} .
$$

Q. E. D.

\footnotetext{
${ }^{6}$ See the appendix for a proof.

${ }^{7}$ See e.g. Maskin and Riley (2000).
} 
Intuitively, even if $\alpha$ has the highest valuation, $\beta$ may win when he is much more risk averse than $\alpha$. Notice that if the bidder with the lowest valuation has the lowest risk aversion level then the auction remains efficient.

Lemma 2 Allocative efficiency decreases as bidders become more asymmetric in terms of risk aversion.

Proof of lemma 2: The higher the right hand side of (??) is, the more inefficient the allocation can be. Consider that both bidders become more asymmetric in terms of risk aversion, i.e. $\rho_{\alpha}$ becomes $\rho_{\alpha}+\varepsilon$ and $\rho_{\beta}$ becomes $\rho_{\beta}-\varepsilon$. Consider also that $v_{\alpha}>v_{\beta}$. Then, the auction is efficient if $\alpha$ wins i.e. if $v_{\alpha}-b_{\alpha}>v_{\beta}-b_{\beta}$. But, bidder $\alpha$ will not win if

$$
\frac{v_{\alpha}-v_{\beta}}{a}<\frac{2\left(1+\rho_{\alpha}+\rho_{\beta}-\sqrt{1+2\left(\rho_{\beta}-\epsilon\right)\left(2+\rho_{\alpha}+\rho_{\beta}\right)}\right)}{2+\rho_{\alpha}+\rho_{\beta}} .
$$

Obviously, the right hand side of (??) is increasing with $\epsilon$.

Q. E. D.

\subsection{The impact of risk aversion levels on expected revenue}

In terms of expected revenue, an interesting question to address is whether the seller's expected revenue increases as bidders become more asymmetric in terms of risk aversion. In order to derive the expected revenue of the seller, assume e.g. that $b_{\alpha} \geq b_{\beta}$. Then we have

$$
\begin{aligned}
E R & =\int_{b_{\alpha}-b_{\beta}+\mu-a}^{a+\mu}\left(v_{\alpha}-b_{\alpha}\right) F\left(v_{\alpha}-b_{\alpha}+b_{\beta}\right) f\left(v_{\alpha}\right) d v_{\alpha} \\
& +\int_{\mu-a}^{a-b_{\alpha}+b_{\beta}+\mu}\left(v_{\beta}-b_{\beta}\right) F\left(v_{\beta}+b_{\alpha}-b_{\beta}\right) f\left(v_{\beta}\right) d v_{\beta} \\
& +\int_{a-b_{\alpha}+b_{\beta}+\mu}^{a+\mu}\left(v_{\beta}-b_{\beta}\right) f\left(v_{\beta}\right) d v_{\beta}
\end{aligned}
$$

Let us consider that $\rho_{\alpha}=\rho+\epsilon$ and $\rho_{\beta}=\rho-\epsilon$, with $0<\epsilon<\min (1-\rho, \rho)$. Then, we have the following proposition ${ }^{8}$

Proposition 2 The seller's expected revenue increases (resp. decreases) as bidders become more asymmetric in terms of risk aversion if

$$
\epsilon>(\text { resp. }<) \frac{-6-5 \rho+4 \rho^{2}+3 \rho^{3}+(2+\rho) \sqrt{9+2 \rho(7+3 \rho)}}{(4+3 \rho)^{2}} .
$$

${ }^{8}$ See the appendix for a proof. 
The result of proposition 2 is in line with proposition 1. Recall that, from proposition 1 , bidder $\beta$ (i.e. the most risk averse bidder) reduces his markup when he becomes more risk averse while his opponent becomes less risk averse (the direct effect dominates the indirect effect). As also stressed by proposition 1 , when asymmetry in terms of risk aversion between both bidders is sufficiently large, bidder $\alpha$ reduces his markup although he becomes less risk averse (while bidder $\beta$ becomes more risk averse); in this case the indirect effect dominates the direct effect. This last point turns out to be crucial on the impact on the seller's expected revenue. Indeed, we can show that when bidders become more asymmetric in terms of risk aversion, the impact on bidding strategies is higher for bidder $\alpha$ than for bidder $\beta$

$$
\frac{\partial\left(b_{\alpha}-b_{\beta}\right)}{\partial \epsilon}=\frac{2 a}{\sqrt{1-4(\epsilon-\rho)(1+\rho)}}>0 .
$$

Thus, the higher the gap between risk aversion levels, the more the indirect effect on strategic markups matters and thus the higher the expected revenue of the seller.

\section{Conclusion}

This paper has considered a first-price sealed-bid auction with two bidders having different relative risk aversion levels. Within a quite realistic informational framework, we have first derived the asymmetric equilibrium bidding strategies. Then we have analyzed the impact on a bidder's strategy of both his own risk aversion and that of his opponent. Highlighting a direct and an indirect effect, we have shown that the less risk averse bidder reduces his markup when he becomes even less risk averse when asymmetry in terms of risk aversion between both bidders is sufficiently large. When this asymmetry is large, we have also shown that the seller's expected revenue increases as bidders become more asymmetric in terms of risk aversion. However, the allocative efficiency of the auction decreases when asymmetry increases.

\section{Appendix}

\section{Proof of proposition 1}

We first show that the direct effect dominates the indirect effect for bidder $\beta$. We can compute 


$$
\frac{\partial b_{\beta}}{\partial \rho_{\beta}}-\frac{\partial b_{\beta}}{\partial \rho_{\alpha}}=-\frac{a\left(-3-\rho_{\alpha}^{2}+\rho_{\alpha}\left(\rho_{\beta}-4\right)+2 \rho_{\beta}\left(1+\rho_{\beta}\right)+A\right)}{A\left(2+\rho_{\alpha}+\rho_{\beta}\right)} .
$$

With $\rho_{\alpha}=\rho+\epsilon$ and $\rho_{\beta}=\rho-\epsilon$, we have

$$
\frac{\partial b_{\beta}}{\partial \rho_{\beta}}-\frac{\partial b_{\beta}}{\partial \rho_{\alpha}}=-\frac{a\left(2(\rho-\epsilon)(1+\rho-\epsilon)-3+(\rho-\epsilon-4)(\rho+\epsilon)-(\rho+\epsilon)^{2}+A\right)}{2(1+\rho) A}
$$

The derivative of the RHS of (??) with respect to $\epsilon$ is equal to

$$
\frac{2 a(3-3 \epsilon(1+\rho)+\rho(7+5 \rho))}{\left(-4 \epsilon(1+\rho)+(1+2 \rho)^{2}\right)^{3 / 2}}
$$

It is straightforward to show that this derivative is positive. Besides, the RHS of (??) evaluated at $\epsilon=0$ is equal to $\frac{a(1-\rho)}{1+2 \rho}$ which is positive. So, $\frac{\partial b_{\beta}}{\partial \rho_{\beta}}>\frac{\partial b_{\beta}}{\partial \rho_{\alpha}}$. Q.E.D.

Consider now the case of bidder $\alpha$. From (??) and (??), we have

$$
\frac{\partial b_{\alpha}}{\partial \rho_{\beta}}-\frac{\partial b_{\alpha}}{\partial \rho_{\alpha}}=a\left(\frac{\epsilon+\rho}{\sqrt{1+2(\rho-\epsilon)(2+2 \rho)}}-\frac{1+\sqrt{1+2(\rho-\epsilon)(2+2 \rho)}}{2+2 \rho}\right) .
$$

Then,

$$
\frac{\partial b_{\alpha}}{\partial \rho_{\beta}}-\frac{\partial b_{\alpha}}{\partial \rho_{\alpha}}>(\text { resp. }<) 0 \text { if } \epsilon>(\text { resp. }<) \frac{1+3 \rho+3 \rho^{2}+\sqrt{1+6 \rho+6 \rho^{2}}}{9(1+\rho)} .
$$

Q.E.D.

\section{Proof of proposition 2}

Substituting $b_{\alpha}$ and $b_{\beta}$ by their values in (??), ER becomes

$$
E R=\mu+\frac{a}{3}\left(\begin{array}{c}
3+\frac{4}{\left(2+\rho_{\alpha}+\rho_{\beta}\right)^{3}}-3 A \\
+\frac{\left(4 A+\rho_{\beta}\left(2+\rho_{\alpha}+\rho_{\beta}\right)\right)}{\left(2+\rho_{\alpha}+\rho_{\beta}\right)^{3}}\left(12+8 A+3 \rho_{\alpha}(1+A)+3 \rho_{\beta}(1+A)\right)
\end{array}\right) .
$$

Consider that $\rho_{\alpha}=\rho-\epsilon$ and $\rho_{\beta}=\rho+\epsilon$, we have

$$
\frac{\partial E R}{\partial \epsilon}=\frac{a(2+2 \epsilon(1+\rho)(4+3 \rho)-2 A-\rho(-3+2 \rho(1+\rho)+A))}{2(1+\rho)^{2} A} .
$$


Then

$\frac{\partial E R}{\partial \epsilon}>0($ resp. $<0)$ if $\epsilon>($ resp. $<) \frac{-6-5 \rho+4 \rho^{2}+3 \rho^{3}+(2+\rho) \sqrt{9+2 \rho(7+3 \rho)}}{(4+3 \rho)^{2}}$

Q.E.D.

\section{References}

[1] Athey, S. And Levin, J., "Information and Competition in U.S. Forest Service Timber Auctions", Journal of Political Economy, Vol 109 (2001), pp. $375-417$.

[2] Biais B. And Bossaerts P., "Asset Prices and Trading Volume in a Beauty Contest", Review of Economic Studies, Vol. 65 (1998), pp. 307-340.

[3] Campo, S., "Risk Aversion and Asymmetry in Procurement Auctions: Identification, Estimation and Application to Construction Procurements", working paper, University of North Carolina at Chapel Hill (2009).

[4] Cox, J., Smith, V. And Walker, J., "Experimental Development of Sealed-Bid Auction Theory Calibrating Controls for Risk Aversion", American Economic Review, Vol. 75 (1985), pp 160-165.

[5] Guerre, E., Perrigne, I. And Vuong, Q .,"Optimal Nonparametric Estimation of First-Price Auctions", Econometrica, vol. 68 (2000), pp. 525-574.

[6] Goeree, J. Holt, C.And Palfrey, T, "Quantal Response Equilibrium and Overbidding in Private Value Auctions", Journal of Economic Theory, Vol 104(1), (2002), pp. 347-472.

[7] Klemperer, P.D., "Auction theory: A guide to the literature". Journal of Economic Surveys, Vol. 13 (1999), pp. 227-286.

[8] Krishna, V., Auction theory, Academic Press, 2002.

[9] Maskin E., And Riley J., "Optimal Auctions with Risk Averse Buyers", Econometrica, Vol. 52 (6) (1984), pp. 1473-1518.

[10] Maskin E., And Riley J., "Asymmetric Auctions", Review of Economic Studies, Vol. 67 (3) (2000), pp. 413-438. 
[11] Matthews S.A., "Selling to Risk Averse Buyers with unobservable Tastes", Journal of Economic Theory, Vol. 30 (3) (1983), pp. 370-400.

[12] Milgrom E., And Weber J., "A Theory of Auctions and Competitive Bidding", Econometrica, Vol. 50 (5) (1982), pp. 1089-1122.

[13] Vickrey W., "Couterspeculation, Auctions and Competitive Sealed Tenders", Journal of Finance, Vol. 16 (1961), pp. 8-37.

[14] Von Ungern-Sternberg T., "Swiss Auctions", Economica, Vol. 58 (1991), pp. 341-357. 